\title{
CHRONIC APICAL PERIODONTITIS IN CHRONIC KIDNEY DISEASE PATIENTS
}

\author{
Georgiana Florentina Moldoveanu ${ }^{1 a}$, Ioana Suciu ${ }^{1 b}$, Paula Perlea ${ }^{1 c}$ \\ 'Department of Endodontology, Faculty of Dental Medicine, "Carol Davila" University of Medicine and Pharmacy, \\ Bucharest, Romania \\ ${ }^{a} \mathrm{MD}$, Assistant Professor \\ ${ }^{b} \mathrm{MD}, \mathrm{PhD}$, Associate Professor \\ 'MD, PhD, Associate Professor
}

Received: April 13, 2016 Received in revised form: April 27,2016

Accepted: May 3, 2016

Published online: April 20, 2016

\section{Cite this article:}

Moldoveanu GF, Suciu I, Perlea P. Chronic apical periodontitis in chronic kidney disease

patients. Stoma Edu J.2016;3(1-2):28-32.

\author{
ABSTRACT \\ https://doi.org/10.25241/stomaeduj.2016.3(1-2).art.4
}

Introduction: The involvement of oral-dental infectious pathology in triggering or worsening kidney diseases has been known for a long time. The purpose of this study was to evaluate the prevalence of chronic apical periodontitis in a group of chronic kidney disease patients and their relationship with the parameters related to renal function.

Methodology: Medical parameters of 51 predialysed adults were extracted from the observation charts of the hospital using a registration form. Chronic apical periodontitis has been diagnosed on periapical radiographs by the presence of any radiolucent areas detected in the apical third of teeth.

Results: $29.41 \%$ of patients had no periapical lesion, $33.33 \%$ had one periapical lesion, $17.64 \%$ had 2 periapical lesions and the remaining $19.62 \%$ had at least three periapical lesions. Chronic apical periodontitis was not statistically significantly associated with any of the demographic variables. Lower serum albumin levels were significantly associated with a greater number of periapical lesions. In addition, the large number of chronic apical periodontitis was significantly associated with high values of cholesterol.

Conclusion: The detection of some abnormal cholesterol and albumin levels during regular investigations of patients with chronic renal failure, require a mandatory dental visits that will be associated with a radiological examination to detect chronic apical periodontitis. The results obtained in our study emphasize the importance of radiological examinations for all patients, prior to kidney transplantation, since they may have teeth with inflammatory lesions, which cannot be clinically detected.

Keywords: chronic apical periodontitis, predialysed patients, cholesterol, albumin.

\section{Introduction}

The oral health of patients with chronic kidney disease (CKD) has become a subject of intense investigation in recent years, not only due to the oral and systemic manifestations of the disease but also due to treatment-related complications. Moreover, the chronic kidney disease is a disease whose incidence is steadily increasing and as a result, a large number of patients are seeking dental care. The possibility that events in the oral cavity may influence systemic diseases has been highlighted by numerous studies on associations and interactions between oral diseases and cardiovascular diseases, myocardial infarction, manifestations during pregnancy, diabetes, and bacterial pneumonia. Research conducted has included epidemiological studies, intervention studies and studies that have attempted to elucidate the mechanisms of action. The results were occasionally contradictory, which is not surprising, given the variations in study designs, populations studied and the statistical analysis used for the studies. ${ }^{1}$ The involvement of oral-dental infectious pathology in triggering or worsening kidney diseases has been known for a long time. ${ }^{2}$ Most evidence gathered involve infectious outbreak, located in the oral cavity, ingravescence of chronic glomerulonephritis with mesangial deposits of $\lg A$, but also in updating and increasing the rate of other renal diseases progression. In this context, the identification of sources of inflammation in patients with CKD and their removal is a matter of great interest in nephrology but also in cardiology 


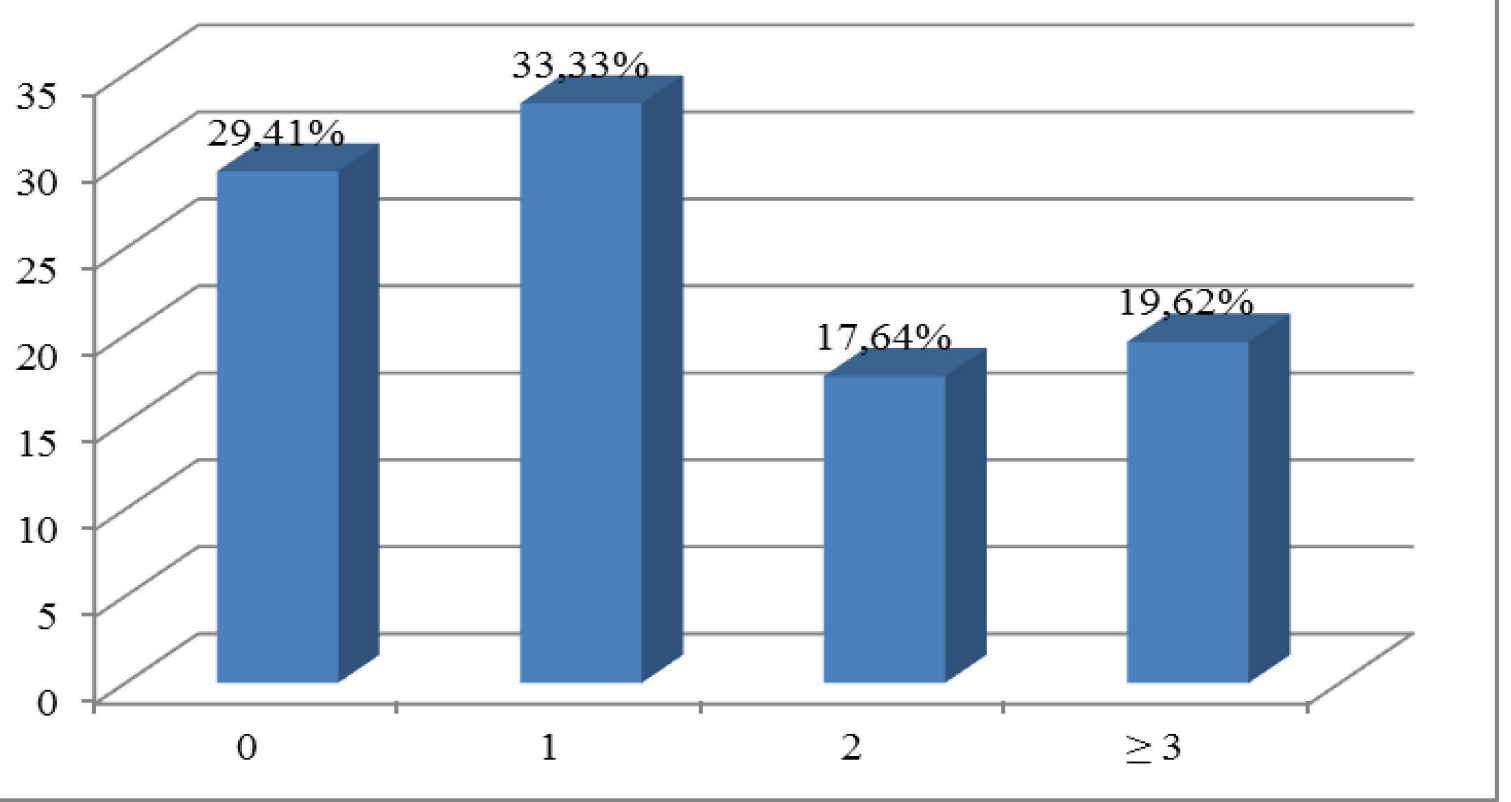

Figure 1. Prevalence of chronic apical periodontitis in the group of patients

and in other specialties.

Apical periodontitis are oral inflammatory disorders associated with systemic inflammatory changes. Endodontic infections are characterized by the presence of some soluble bacterial products with a strong pro-inflammatory potential. Lipopolysaccharides, present and released from the gram-negative cell walls, are components of endodontic bacteria best described to induce cytokines and other inflammatory components as IFN $\gamma$, IL- $1 \alpha$ and $\beta$, IL6, IL 8, TNF $\alpha{ }^{3}$

The purpose of this study was to evaluate the prevalence of chronic apical periodontitis in a group of chronic kidney disease patients and their relationship with the parameters related to the renal function.

\section{Materials and methods}

51 predialysed adults were included in this study that was approved by the Ethical Committee of the "Carol Davila" University of Medicine and Pharmacy, number 31 on August 9, 2014. They were known for at least one year to have CKD (GFR $<60 \mathrm{ml} / \mathrm{min} / 1.73 \mathrm{~m}^{2}$ ), had the ability to understand the protocol and signed the informed consent form. Bimaxillary edentulous patients under immunosuppressive treatment, those with mental retardation, mental illness or malignancy, pregnant or lactating women were excluded.

The medical parameters were extracted from the observation charts of the hospital using a registration form that included: age, gender, residence, education level, smoker/non-smoker status, the diagnosis of basic kidney disease, current level eGFRs, the stage of CKD, association or not with diabetes or not, acid-base balance parameters of bone mineral metabolism, anthropometric and biochemical parameters of nutritional status, hematological changes and state of systemic inflammation. Panoramic radiographs were performed to diagnose chronic periapical dental infections or other pathology existing in the bone tissue. Retroalveolar X-rays were indicated by the contributor specialist radiologist after seeing the orthopantomograms, for those cases where he considered them to correctly diagnose chronic apical periodontitis. Chronic apical periodontitis has been diagnosed by the presence of any radiolucent areas detected in the apical third of teeth. The diagrams were made using Microsoft Excel 2007, and the statistical analysis was performed using SPSS statistical analysis software version 19. Statistical correlations between variables were tested by using the tool Analyze $\rightarrow$ Correlate $\rightarrow$ Bivariate.

\section{Results}

Chronic apical periodontitis was diagnosed radiographically and confirmed by the presence of any radiolucent areas detected in the apical third of teeth. Thus, $29.41 \%$ of patients had no periapical lesion, $33.33 \%$ had one periapical lesion, $17.64 \%$ had 2 periapical lesions and the remaining $19.62 \%$ had at least three periapical lesions (Fig. 1). Chronic apical periodontitis were not statistically significantly associated with any of the demographic variables (Table 1).

In exchange, lower serum albumin levels were significantly associated with a greater number of periapical lesions. In addition, the large number of chronic apical periodontitis was significantly associated with high values of cholesterol. There was evidenced no significant relationship between chronic apical periodontitis on the one hand, and smoking, diabetes or body mass index (BMI), on the other hand (Table 2). The presence of apical lesions was not associated with any estimated glomerular filtration rate, or the chronic kidney disease stage (Table 3). 
Table 1. Relations between apical lesions and demographic variables

\begin{tabular}{|c|c|c|c|c|c|}
\hline & Gender & Age & Environment & Education & CKD Etiology \\
\hline Apical Lesions & & & & & \\
Pearson Correlation & .199 & -.038 & .083 & -.141 & -.095 \\
Sig.(2-tailed) & .162 & .791 & .562 & .322 & .509 \\
$\mathrm{~N}$ & 51 & 51 & 51 & 51 & 51 \\
\hline
\end{tabular}

Table 2. Relationship between chronic apical periodontitis on the one hand, and smoking, diabetes, $\mathrm{BMI}$, cholesterol, serinemia, on the other hand

\begin{tabular}{|c|c|c|c|c|c|}
\hline & Smoking & Diabetes & BMI & Cholesterol & Albumin \\
\hline Apical lesions & & & & & \\
Pearson Correlation & .119 & -.199 & -.010 & $.411^{\star \star}$ & $-.440^{\star \star}$ \\
Sig.(2-tailed) & .404 & .162 & .948 & .004 & .002 \\
N & 51 & 51 & 48 & 48 & 48 \\
\hline
\end{tabular}

**. The association is significant at $p<0.01$

Table 3. Relations between apical lesions on the one hand, and eRFG, CKD stage, on the other hand

\begin{tabular}{|c|c|c|}
\hline & eRFG & CKD Stage \\
\hline Apical lesions & & \\
Pearson Correlation & .034 & .005 \\
Sig.(2-tailed) & .813 & .972 \\
N & 50 & 50 \\
\hline
\end{tabular}

\section{Discussion}

The study was performed on a group of 51 patients, the same number used by Buhlin et al, ${ }^{4}$ who investigated the oro-facial health of patients with end-stage renal disease, focusing on their periodontal conditions. They showed that a substantial number of patients who suffer from chronic kidney disease have dental problems that required attention.

The investigation of infection sources in this study was complemented by panoramic radiographs, which enabled visualization of teeth together with the bone support structures. Studies using radiological analysis for patients with chronic kidney disease are limited in the literature.

In the present study, the most common radiological changes observed were deposits of calculus, dental caries and the presence of chronic apical periodontitis. For the latter, periapical radiographs were the only means of identification, approximately $70 \%$ of patients from the group investigated having at least one periapical lesion, characteristic for chronic apical periodontitis. In a similar study, Thorman et al. ${ }^{5}$ compared the panoramic radiographs of 93 pre-dialytic and dialytic patients with chronic kidney disease with a control group and found an increased prevalence of periapical infections in patients from the study group.

Epidemiological studies have shown that apical periodontitis is a chronic common disease in the general population. $6,7,8$ However, data on the prevalence of chronic apical periodontitis vary between populations and countries, and depend on differences in the prevalence of dental caries, access to dental services and the methodology used.

Thus, a study conducted in Portugal on a group of 322 individuals resulted in a $27 \%$ prevalence of chronic apical periodontitis ${ }^{6}$ and another study in Norway a $16 \% .^{7}$ Higher values were reported in research conducted by Jiménez-Pinzón et al. in Spain (61.1\%), ${ }^{8}$ Loftus et. al in Ireland (33.1\%), ${ }^{9}$ Tsuneishi et al. ${ }^{10}$ in Japan (69.8\%), Demo et al. ${ }^{11}$ in Belgium (63.1\%).

The results of this study showed that elevated serum cholesterol levels (> $190 \mathrm{mg} / \mathrm{dL}$ ) are associated with an increased number of chronic apical periodontitis.

The explanation could be given by the presence of cholesterol crystals, commonly found in biopsies of periapical lesions. It seems these crystals come from disintegrated erythrocytes present in the blood vessels stagnant inside a lesion, lymphocytes, plasma cells and macrophages (which decay into periapical lesions) and circulating plasma lipids. Once they have been deposited, cholesterol crystals act as irritants and cause foreign body reactions. Macrophages and giant cells trying to devour cholesterol crystals, but are unable to degrade crystalline cholesterol. Furthermore, macrophages exposed to cholesterol crystals apparently act like a bone lysis and thus a chronic inflammation in the periapical area is supported. ${ }^{12}$

Cholesterol is also related to another common 
condition in the chronic kidney disease, the cardiovascular disease. Atherosclerosis is a multifactorial disease, which occurs at young ages and evolves with age. The presence of cholesterol crystals is also recognized in atherosclerotic plaques in humans.

Slutzki-Goldberg et al. ${ }^{13}$ conducted a study in which they evaluated the possible link between age and cholesterol deposits in periapical lesions. They have examined the biopsies of lesions identified in a group of teenagers and one of elderly and found that there is a significantly increased incidence of cholesterol deposits in periapical biopsies performed in elderly individuals, where serum cholesterol levels were elevated. The cholesterol crystal formation mechanism is probably similar to the genesis of atherosclerosis.

Chronic apical periodontitis is an acute or chronic inflammatory lesion, present around the apex of a tooth and caused by a bacterial infection of the endodontic system.

Histologically, it is represented by a periapical inflammatory response that occurs after the resorption of the adjacent support bone and local infiltration of inflammatory cells. ${ }^{14}$

Thus, the significant associations, between lower serum albumin values and the high number of periapical lesions, from this study were not statistically surprising given that hypoalbuminemia ( $<3.5 \mathrm{mg} / \mathrm{dL}$ ) can be caused by acute or chronic inflammatory responses.

\section{Conclusions}

This descriptive study showed that chronic apical periodontitis is highly prevalent in patients with chronic kidney disease, but future research is required to determine whether endodontic diseases are worsened by the chronic kidney disease or vice versa.

The detection of some abnormal cholesterol and albumin levels during the regular investigations of patients with chronic renal failure, require a mandatory dental visits that will be associated with a radiological examination to detect chronic apical periodontitis. Most often silent from a clinical point of view, chronic apical periodontitis is diagnosed accidentally or following an acute exacerbation. These are powerful outbreaks of infection with diverse microbial flora camped at this level, able to cause amplification of the inflammatory response in the chronic kidney disease.The results obtained in our study emphasize the importance of radiological examinations for all patients, prior to kidney transplantation, since they may have teeth with inflammatory lesions, which cannot be clinically detected.

According to the World Health Organization, health is an essential part of the social, economic and personal development of the individual and an important component of the quality of life. Especially in patients with CKD, the health of the oral cavity must be permanently assisted and improved in order to maintain a good overall body condition.

\section{Acknowledgments}

The authors declare no conflict of interest related to this study. There are no conflicts of interest and no financial interests to be disclosed.

\section{REFERENCES}

1. Barnett ML. The oral-systemic disease connection. An update for the practicing dentist. J Am Dent Assoc. 2006;137 Suppl 2:5S-6S.

2. Ursea N. Insuficiența renală cronică. In: Esențialul în nefrologie, Ursea N, editor. Bucureşti: Editura Fundației Române a Rinichiului; 2000:197-226.

3. Gomes MS, Blattner TC, Filho MS, Grecca FS, Hugo FN, Fouad AF, Reynolds MA. Can apical periodontitis modify systemic levels of inflammatory markers? A systematic review and meta-analysis. J Endod. 2013;39(10):1205-1217.

4. Buhlin K, Bárány P, Heimbürger 0 , Stenvikel P, Gustafsson A. Oral health and pro-inflamatory status in end-stage renal disease patients. Oral Health Prev Dent. 2007;5(3):235244.

5. Thorman R, Neovius M, Hylander B. Clinical findings in oral health during progression of chronic kidney disease to end-stage renal disease in a Swedish population. Scand J Urol Nephrol. 2009;43(2):154-159.

6. Marques MD, Moreira B, Eriksen HM. Prevalence of apical periodontitis and results of endodontic treatment in an adult, Portuguese population. Int Endod J. 1998;31(3):161165.

7. Skudutyte-Rysstad R, Eriksen HM. Endodontic status amongst 35-year-old Oslo citizens and changes over a 30 year period. Int Endod J. 2006;39(8):637-642.

8. Jiménez-Pinzón A, Segura-Egea JJ, Poyato-Ferrera $M$,
Velasco-Ortega E, Ríos-Santos JV. Prevalence of apical periodontitis and frequency of root-filled teeth in an adult Spanish population. Int Endod J. 2004;37(3):167-173.

9. Loftus JJ, Keating AP, McCartan BE. Periapical status and quality of endodontic treatment in an adult Irish population. Int Endod J. 2005;38(2):81-86.

10. Tsuneishi M, Yamamoto T, Yamanaka R, Tamaki N, Sakamoto T, Tsuji K, Watanabe T. Radiographic evaluation of periapical status and prevalence of endodontic treatment in an adult Japanese population. Oral Surg Oral Med Oral Pathol Oral Radiol Endod. 2005;100(5):631-635.

11. De Moor RJ, Hommez GM, De Boever JG, Delmé KI, Martens GE. Periapical health related to the quality of root canal treatment in a Belgian population. Int Endod J. 2000;33(2):113-120.

12. Nair PN. On the causes of persistent apical periodontitis: a review. Int Endod J. 2006;39(4):249-281.

13. Slutzky-Goldberg I, Baev V, Volkov A, Zini A, Tsesis I. Incidence of cholesterol in periapical biopsies among adolescent and elderly patients. J Endod. 2013;39(12):1477. 1480 .

14. Segura-Egea JJ, Jimenez-Moreno E, Calvo-Monroy C, Ríos-Santos JV, Velasco-Ortega E, Sánchez-Domínguez B, Castellanos-Cosano L, Llamas-Carreras, JM. Hypertension and dental periapical condition. J Endod. 2010;36(11):1800-1804. 


\section{Georgiana Florentina MOLDOVEANU}

MD, Assistant Professor

Department of Endodontology, Faculty of Dental Medicine,

"Carol Davila" University of Medicine and Pharmacy, Bucharest, Romania

Dr. Georgiana Florentina Moldoveanu graduated in 2005 from the Faculty of Dental Medicine, "Carol Davila "University of Medicine and Pharmacy, Bucharest, Romania. She is a specialist in Dental Surgery and Endodontics and currently a teaching assistant in the Department of Endodontology. In addition to her teaching activities, Dr. Moldoveanu runs a part-time private practice in Bucharest.

Her research focuses on endodontic retreatment, restoration of endodontically treated teeth and treatment of apical periodontitis.

She is a member of the European Society of Endodontology and the Romanian Association of Endodontology.

\section{Orestions}

\section{How many patients were included in the study?}

$\square$ a.24;

b. 30;

口c. 51;

ad. 78 .

\section{What percentage of the group had more than 3 apical lesions?}

a. $22 \%$;

b. $19.62 \%$;

c. $29,41 \%$;

d. $50 \%$.

\section{How was chronic apical period ontitis diagnosed?}

$\square$ a. Cone beam computed tomography;

$\square$ b. Bitewing radiographs;

c. Panoramic radiographs;

$\checkmark$ d. Periapical radiographs.

\section{Which of the following is false?}

$\square$ a. Chronic apical periodontitis was not statistically significantly associated with any of the demographic variables;

$\square$ b. Lower serum albumin levels were significantly associated with a greater number of periapical lesions;

$\square$ c. The large number of chronic apical periodontitis was not significantly associated with high values of cholesterol;

$\square$ d. The presence of apical lesions was not associated with any estimated glomerular filtration rate, or the chronic kidney disease stage. 